\title{
Acknowledgment to Reviewers of Methods and Protocols in 2021
}

\author{
Methods and Protocols Editorial Office
}

Citation: Methods and Protocols

Editorial Office. Acknowledgment to Reviewers of Methods and Protocols in 2021. Methods Protoc. 2022, 5, 12. https://doi.org/10.3390/mps5010012

Published: 24 January 2022

Publisher's Note: MDPI stays neutral with regard to jurisdictional claims in published maps and institutional affiliations.

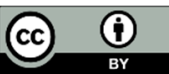

Copyright: $(2022$ by the authors. Licensee MDPI, Basel, Switzerland. This article is an open access article distributed under the terms and conditions of the Creative Commons Attribution (CC BY) license (https://creativecommons.org/licenses/by/4.0/)
MDPI AG, St. Alban-Anlage 66, 4052 Basel, Switzerland

Rigorous peer-reviews are the basis of high-quality academic publishing. Thanks to the great efforts of our reviewers, Methods and Protocols was able to maintain its standards for the high quality of its published papers. Thanks to the contribution of our reviewers, in 2021, the median time to first decision was 25 days and the median time to publication was 42 days. The editors would like to extend their gratitude and recognition to the following reviewers for their precious time and dedication, regardless of whether the papers they reviewed were finally published:

\begin{tabular}{|c|c|}
\hline Adel Martinez-Martinez & Camelia Quek \\
\hline Ahmed Hieawy & Camilla Pierella \\
\hline Alberto Lo Gullo & Carina Bernardo \\
\hline Aleksandra Kołota & Carmen Mirabelli \\
\hline Alessandro A. Sartori & Caterina Maria Gambino \\
\hline Alexandre Barbosa & Chih-Wei Pai \\
\hline Alexey Bogdanov & Christopher Hellen \\
\hline Alisson Aliel Vigano Puglies & Corinna Geisler \\
\hline Amani Gillette & Corinna Preusse \\
\hline Amulya Yaparla & Cosimo Nardi \\
\hline Anahid Ahmadi Birjandi & Cristian Iulius Surcel \\
\hline Anastas Gospodinov & Dafne Campigli Di Giammartino \\
\hline András Bikov & Daniela Gradia \\
\hline Andrej Besse & Daria Dziewulska \\
\hline Andrew Somogyi & Debadrita Paria \\
\hline Aneta Wieczorek & Denis Bourgeois \\
\hline Anette Gjörloff Wingren & Denise Corridore \\
\hline Anita Turner & Dhiman Pal \\
\hline Anoop Sheshadri & Diego San Mauro \\
\hline Anthony J. Baucum & Dinesh Jillella \\
\hline Aravindhan Ganesan & Eduardo Oliver \\
\hline Areti Stavropoulou & Edyta Pasicka \\
\hline Armando Villalta & Ekaitz Errasti-Murugarren \\
\hline Armin Ensser & Elizabeth Johnson \\
\hline Arunava Ghosh & Elmer Maurits \\
\hline Atsuo Yoshido & Emer Ferro \\
\hline Badreddine Douzi & Emma Siesmaa \\
\hline Barbara Ruaro & Eric Vachon \\
\hline Béla Kovács & Erwin De Bruin \\
\hline Bruno Chrcanovic & Ewa Sadowy \\
\hline Bruno Rizzuti & Ewa Tomaszewska \\
\hline Calum McHale & Ewelina Zielińska \\
\hline
\end{tabular}


Faisal Jamil

Federica Fogacci

Gerald Chi

Gerard Lee L. See

Gerardo Pellegrino

Giorgio Lombardo

Goran Muric

Grzegorz Przybylski

Guoqing Wang

Gutemberg Alves

Guy K. German

Hamada Elwan

Han Su Kim

Heike Rudolph

Henna Muzaffar

Henri Vahabi

Hervé Reychler

Hongfu Yuan

Ian James Martins

Ieva Meidute-Kavaliauskiene

Ivica Pelivan

Jacek Bogusław Szmańda

Jacinto Jardim

Jadwiga Hamułka

Jakob Morgenstern

Jan Koster

Janja Trcek

Jiaoju Ge

Joanna Sztuba-Solinska

João Pinheiro

Joaquin De Navascues

Joe Millward

John A. Kalef-Ezra

Jong Kook Park

José A. Jiménez Heffernan

Ju Hyun Park

Juergen Reichardt

Jun Ying Li

Junchul David Yoon

Justyna Godos

Karin G. F. Gerritsen

Karsten Gülow

Karsten Schrobback

Katarzyna Kuter

Katarzyna Otulak

Kátia Cândido Carvalho

Kazunari Yoshida

Kazushige Oshita

Kelvin Ian Afrashtehfar

Khaled Elsayed Ahmed

Konstantin Chekanov
Kumar Saurav

Lachlan H. Thompson

Laleh Yerushalmi

Laurence J. Walsh

Laurent Corcos

Leonard L. Dobens

Lidia Sabater

Liron Klipcan

Lorena Pochini

Lucía Monaco

Magdalena Baymakova

Małgorzata Duda

Manuel Sanchez-Martin

Maria Angela Pellegrino

Maria M. Ulaszewska

Maria Pina Serra

Maria Spletter

Marianna D'anca

Marianna Kapetanou

Marianna Tzanoudaki

Mario Zurita

Martin Giurfa

Mary Lou Cutler

Massimo Libra

Mauro Castro

Michelle M. W. Feijen

Mikhail A. Liskovykh

Ming-Lun Hsu

Mirza Pojskic

Moses Ayoola

Murielle Ålund

Natalia Cheshenko

Nicola Ramacciati

Ning Zhao

Nishikant Wase

Pablo Luna-Nevarez

Pallabi K. Pal

Paolo Calligari

Paolo Capparè

Patricia Kitsao-Wekulo

Peter Esser

Ping-Chieh Pao

Piotr Wychowański

Pradeep Dagur

Qi Yu

R. Brent Seale

Rachael Crew

Radoslaw Bednarek

Raif Yuecel

Reema Singh

Regina Ebert 
Renato Gondar

Rita Hõrak

Rosângela Schwarz Rodrigues

Rukshan Mehta

Salvatore Bocchieri

Sébastien Lamy

Shannon Gray

Shumit Saha

Steven Vollmer
Susan Mirlohi

Svetla Baykoucheva

Takahito Nakajima

Tatiana S. Frolova

Toshiyuki Takahashi

Tsutomu Nakazawa

Wenyang Dong

Yong Ge

Yun Han 